\title{
Perceived barriers to goals of care discussions in the emergency department: a multi-center analysis of hospitalist physicians
}

\section{Vanessa Zannella}

University of Toronto

Kieran Quinn ( $\Delta$ kieran.quinn@sinaihealth.ca )

Sinai Health System

\section{Samuel Vaillancourt}

St. Michael's Hospital

Jonathan Ailon

St. Michael's Hospital

Melissa Mcgowan

St. Michael's Hospital

\section{Niran Argintaru}

University of Toronto

\section{Research Article}

Keywords: Palliative care, goals of care, emergency department, hospitalist, internal medicine

Posted Date: January 8th, 2021

DOl: https://doi.org/10.21203/rs.3.rs-137260/v1

License: (1) This work is licensed under a Creative Commons Attribution 4.0 International License.

Read Full License 


\section{Title Page:}

Manuscript Title:

Perceived barriers to goals of care discussions in the emergency department: a multi-center analysis of hospitalist physicians.

Author list and affiliations:

Zannella VE 1, Vaillancourt S 1.24, Argintaru N 1, McGowan M 2, Ailon J 3, Quinn KL ${ }_{1.56}$

1 Department of Medicine, University of Toronto, Toronto, Ontario, Canada.

2 Department of Emergency Medicine, St. Michael's Hospital, Toronto, Ontario, Canada.

3 Department of Medicine, St. Michael's Hospital, Toronto, Ontario, Canada.

4 Li Ka Shing Knowledge Institute, St. Michael's Hospital, Toronto, Canada.

5 Department of Medicine, Sinai Health System, Toronto, Ontario, Canada.

6 Institute of Health Policy Management and Evaluation and Department of Medicine, University of Toronto, Toronto, Ontario, Canada.

Corresponding Author:

Dr. Kieran L Quinn

Sinai Health System, University of Toronto

60 Murray Street, $2^{\text {nd }}$ Floor Room 404

Toronto, Ontario, Canada, M5T 3L9

416-843-8403

Kieran.quinn@sinaihealthsystem.ca

Running Title: GOC discussions in the ED

Number of references: 20

Number of tables: 2

Number of figures: 1

Number of appendices: 3

Full word count: 2500

Abstract word count:298

Key words: Palliative care, goals of care, emergency department, hospitalist, internal medicine 


\section{Abstract:}

Background: Patients who present to hospital require clarification and confirmation of their goals of care in the emergency department to align treatment recommendations with their stated goals. The potential barriers to having these discussions in this setting are not well described. The objective of this study was to identify the most substantial barriers to conducting goals of care discussions in the emergency department as reported by hospitalist physicians who are often the first physician that a patient encounters during their admission to hospital.

Design: A team of physicians from Palliative Care, Hospitalist Medicine and Emergency Medicine modified an existing self-reported survey that explored three domains of potential barriers to goals of care discussions: patient-related factors, environmental and resource-related factors, and factors related to physician responsibility and training. Across these domains, hospitalist physicians from 20 hospitals in the Greater Toronto Area in Ontario, Canada were invited to respond on a likert-scale to 17 questions that explored potential barriers to goals of care discussions between January 2018 and April 2019. Responses were rank-ordered by the magnitude of the differences between those that agreed with a statement and those that disagreed.

Key Results: A total of 181 physicians from 17 institutions were included in the study (110 staff physicians, 71 resident physicians, response rate $36 \%$ ). Ninety-five percent of physicians reported engaging in goals of care discussions at least several times per month. The most substantial barriers to goals of care discussions in the emergency 
department reported by hospitalists were: difficulty communicating with outpatient healthcare providers, the emergency department environment, and the lack of prognostic tools for patients with terminal noncancer illness.

Conclusions: Several modifiable barriers to conducting goals of care discussions in the emergency department exist, which were primarily related to difficulty with communication, prognosis and a challenging care environment. 


\section{Background and Rationale:}

The Emergency Department (ED) is the first point of contact for patients requiring urgent admission to hospital. Initial conversations in the ED that elicit a patient's goals of care $(\mathrm{GOC})$ are important because they often influence their subsequent hospital care. GOC discussions involve communication between clinicians and their patient to develop a shared understanding of a patient's health status, values and goals. Their aim is to establish a care plan that is in alignment with the patient or their substitute decision maker's wishes $(1,2)$. While studies show that many patients think about their preferences for end-of-life care, few report having discussions about them with their clinicians (3). A gap in the delivery of goal-concordant care in hospitalized patients may therefore be partially related to challenges in clarifying patients' goals early in their hospitalization. Since the most intense treatments occur immediately in the ED when a patient is the sickest, it is imperative to clarify GOC early in the ED to prevent a patient from receiving treatments that are misaligned with their underlying values or preferences for care. Seventy percent of older adults who are hospitalized in Canada report goals of care that focus on providing comfort rather than life-prolonging treatment, yet $54 \%$ of these patients are admitted to intensive care units at the end of life (4), likely attributed to a lack of early GOC discussions.

Studies examining the challenges to having GOC discussions in the ED are limited. However, evidence demonstrates that GOC discussions lead to better quality of life and reduced costs near the end of life (5). A recent study of 130 Emergency Medicine physicians reported that the perceived barriers to having GOC discussions in 
the ED were related to time constraints, environmental factors and patient expectations (6). Other studies noted that lack of physician training and comfort were potential barriers to conducting GOC discussions in the ED. Physicians often perceive these conversations as challenging, lack confidence in their own communication skills and experience emotional distress while having these conversations (7-10).

Hospitalist physicians provide a large portion of care to hospitalized patients and play an integral role in defining the care trajectory for their patients, which often begins with initial goals of care discussions in the ED $(6,11,12)$. A better understanding of these potential barriers may help identify areas for improvement early on in a patient's hospital trajectory. A large multicentered study of barriers to GOC discussions among seriously ill inpatients suggested patient and family-related factors as the most important barriers (12), but the study was not specifically in the ED where aggressive treatments are most often initiated. Studies that assess potential barriers to goals of care discussions in the emergency department as reported by Hospitalist physicians are lacking. The objective of this study was to identify the most important barriers to conducting goals of care discussions in the emergency department as reported by hospitalist physicians.

\section{Methods:}

\section{Study Setting and Population}

We conducted a multi-center, survey-based study aimed at identifying perceived barriers to having GOC discussions in the emergency department for patients who were referred to the hospitalist service and required hospitalization. We invited 504 practicing hospitalist and resident physicians (270 staff physicians and 234 resident physicians) 
from 20 academic and community hospitals in the Greater Toronto Area in Ontario, Canada, to participate between January 2018 and April 2019. Resident physicians were required to be currently enrolled in an accredited Internal Medicine residency training program at the University of Toronto to be eligible to participate.

\section{Survey Design and Dissemination}

A self-reported survey examining the barriers to goals of care discussions for patients in the Emergency Department was modified based on a previously developed survey (6). The survey consisted of multiple choice, Likert-scale and open-ended questions. A team of experts comprised of faculty with specialty training in Emergency Medicine (EM), Palliative Care, Hospitalist medicine and a physician with dual EM and Palliative Care certification, originally developed the survey to measure the perceived barriers to GOC discussion as reported by ED physicians (6). The survey was subsequently modified by the same team of experts to address some of the specific issues potentially faced by hospitalist medicine physicians. The survey questions covered 3 key domains related to having GOC discussions: 1) patient related factors (4 questions), 2) physician responsibility and training (4 questions), and 3) emergency environment and resources (9 questions). A single open-ended question was also included to capture potential themes not directly addressed by the survey.

Hospitalist physicians and residents were invited to complete the survey through an email containing an electronic link (SurveyMonkey, Palo Alto, CA), or by using paper copies of the survey which were distributed during rounds, meetings, or seminars.

Reminders to complete the survey were sent at day 10,21 and 50 . 


\section{Outcomes}

The primary outcome was the barriers to having GOC discussions in the ED. The secondary outcome was the facilitators to having GOC discussions in the ED. Questions were deemed to be a potential barrier (preventing GOC discussions) or facilitator (promoting GOC discussions) based on consensus by the expert research team. For example, being able to access comprehensive patient records before conducting goals of care discussions was not seen as a potential barrier, but rather a potential facilitator.

\section{Analysis}

Descriptive statistics were used for all multiple choice and Likert-scale questions. The 5 response categories were combined to create three discrete categories for each question: 'almost never' with 'never' ('never'), 'strongly disagree' with 'disagree' ('disagree'), 'sometimes' with 'neutral' ('neutral') and 'almost always' with 'always' ('always') or 'strongly agree' with 'agree' ('agree'). We calculated the difference in the proportion of participants who responded 'always' and 'never', and between 'disagree' and 'agree'. The magnitude of these differences were rank ordered to create a list of the most important barriers or facilitators. The reason for calculating the magnitude of these differences was to statistically test each question for a significant difference, using the null hypothesis that all responses would be neutral and that there would be no significant difference. By doing so, we were able to measure the strength of response over conventional rank order methods that create hierarchy. Responses with a large 
magnitude of difference but that were not barriers based on the directionality of the response related to the question were excluded from the rank order list (7 questions). A secondary analysis was performed to identify the most important barriers or facilitators within each of the 3 domains by placing each survey question into its respective domain. The magnitude of the differences were rank ordered against the other responses within each domain. Barriers were also categorized as modifiable versus non-modifiable based on consensus by the expert research team.

The magnitude of the differences for each question were compared using a Chisquare test to test for a statistically significant difference, using the null hypothesis of no difference. Two independent members of the team reviewed the responses to the openended question. Representative statements were selected for each domain based on consensus.

All quantitative analyses were performed using Microsoft Excel, version 2016 (Redmond, WA).

Ethics approval and consent to participate

Ethics approval for this study was obtained from the Research Ethics Board at St. Michael's Hospital.

\section{Results:}

One hundred eighty-one physicians ( $61 \%$ staff, $n=110,39 \%$ residents, $n=71)$ from 17 hospitals ( $n=11$ community, $n=6$ academic) participated in the survey (overall response rate $36 \%)$. 


\section{Baseline Participant Characteristics}

Physician demographics are reported in Table 1 . The majority of staff physicians were male $(70 \%, n=77)$, with $0-5$ years of practice experience $(43 \%, n=47)$. There was an equal proportion of resident physicians who were male/female ( $49 \%$ male, $n=35)$, which was represented across all training years. $95 \%$ of all physicians reported having GOC discussions in the ED at least several times per month.

\section{Barriers to GOC Discussions in the ED}

The most important barriers to having GOC discussions in the ED were: difficulty communicating with patient's healthcare providers $(79 \%$ difference, $p<0.001)$, the ED working environment $(72 \%$ difference, $p<0.001)$ and the lack of prognostic tools for patients with non-cancer related terminal disease ( $58 \%$ difference, $p<0.001)$ (Figure 1). All barriers were within the domains related to the ED environment (6/10 barriers) and patient-related factors (4/10 barriers), which are rank ordered in Figure 1. No barriers were identified within the domain of physician training and responsibility. Representative responses of hospitalist physicians to the prompt, 'What do you find challenging about having Goals of Care Discussions in the ED?', are shown in Table 2.

\section{Modifiable Barriers to GOC Discussions in the ED}

We identified modifiable barriers to GOC discussions as: the ED working environment, time, prognostic tools, communication with families, substitute decision 
makers and healthcare providers and the availability of palliative care services. The highest ranked modifiable barrier was difficulty communicating with outside healthcare providers.

\section{Facilitators to GOC Discussions in the ED}

Physicians reported that they felt comfortable $(26 \%$ difference, $p=0.005)$ and adequately trained $(47 \%$ difference, $p<0.001)$ to have GOC discussions. Moreover, physicians agreed that GOC discussions were within their scope of practise $(98 \%$ difference, $p<0.001$ ) and they were able to access comprehensive patient records to inform these discussions ( $46 \%$ difference, $p<0.001$ ). For residents of long term care facilities who are mandated to have an advanced directive, physicians reported this directive was available ( $49 \%$ difference, $p<0.001)$, but still believed GOC discussions were necessary in the ED $(75 \%$ difference, $p<0.001)$. Physicians reported mixed results about whether they were more comfortable having GOC discussions for patients with cancer versus patients with noncancer related terminal illness $(6 \%$ difference, $p<0.001)$.

\section{Discussion:}

This multicenter survey of Hospitalist physicians in Ontario, Canada identified that difficulty communicating with outpatient healthcare providers, the emergency department environment, and the lack of prognostic tools for patients with terminal noncancer illness were the most important barriers to conducting GOC discussions in the ED. These findings are important because hospitalist physicians are often one of the 
first physicians that a patient encounters during their admission to hospital and play a crucial role in defining the type of care that hospitalized patients will subsequently receive.

While continued education and capacity building for physician competency in GOC discussions are important, our findings suggest that environmental modifications may also be an area of focus to overcome the barriers identified in this study. Specifically, improvements in the often-chaotic ED working environment, ongoing time pressures, lack of prognostic tools for specific subsets of patients, and facilitated communication with families, substitute decision makers and healthcare providers may serve to circumvent some of these modifiable barriers. In clinical practise, hospitalists may focus on building a more ideal space within the ED for these discussions (e.g. dedicated private and quite GOC rooms) and can advocate for these discussions to take place prior to coming to the ED as part of routine care with outpatient providers.

Our study is the first to describe barriers reported by Hospitalist physicians for patients in the ED. A study of 130 EM physicians found that the top-rated reported barriers to GOC discussions in the ED were related to the ED environment (e.g., time, inability to reach substitute decision maker) or patient experience (e.g., lack of relationship with the patient, patient expectations)(6). A separate study of 1,256 hospitalist physicians reported that the top-rated barriers to GOC discussions for hospitalized patients on inpatient wards were patient and family member-related factors (e.g., difficulty accepting a poor prognosis, understanding the limitation and complications of life-sustaining treatments and disagreement between family members) (12). Our study reinforces these prior findings that the ED environment and patient- 
related factors play a substantial role as potential barriers to GOC discussions in the ED, where critical and often aggressive therapies are initiated.

The strengths of our study are that it is a multicenter survey of staff and resident physicians practicing in multiple institution that are both academic and community based, which serves to enhance the study's generalizability. The limitations of our study are that it was conducted in an urban centre, in a publicly- funded healthcare system where Hospitalists are actively involved in the care of patients in the ED. These practices may vary somewhat in other settings such as rural centres, different institutional practices where Hospitalist physicians may not be involved in the admission process from the ED, or in countries where training of Hospitalist physicians differ. The self-reported voluntary nature of our study also makes it subject to recall and response biases. Lastly, our response rate of $37 \%$ may reflect an element of selection bias within our study sample. However, this rate compares well with similar survey-based studies $(13,14)$.

Our results highlight modifiable and non-modifiable barriers and establish a rationale to focus future studies aimed at mitigating these barriers. Prior studies suggest that a shift from disease-centered, to patient goals-directed, care may be associated with decreased unwanted healthcare $(15,16)$. A dedicated emergency department 'palliative care pathway' may help clarify goals of care for patients with serious illness who present to the ED where treatment can prioritize distressful symptoms above treating physiologic abnormalities for patients with a poor prognosis (17-20). 


\section{Conclusion}

Several modifiable barriers to conducting goals of care discussions in the emergency department exist, which were primarily related to difficulty with communication, prognosis and a challenging care environment.

\section{Declarations:}

\section{Ethics approval and consent to participate}

Ethics approval for this study was obtained from the Research Ethics Board at St. Michael's Hospital. Informed consent was obtained from all participants and all methods were carried out in accordance with relevant guidelines and regulations.

\section{Consent for publication}

Informed consent was obtained from all participants for publication. The results/data/figures in this manuscript have not been published elsewhere and are not under consideration for publication elsewhere.

\section{Availability of data and materials}

The datasets used and/or analyzed during the current study are available from the corresponding author on reasonable request.

\section{Competing interests}

The authors have no competing interests as defined by $\mathrm{BMC}$, or other interests that might be perceived to influence the results and/or discussion reported in this paper.

\section{Funding}

There was no funding required to complete this paper. 


\section{Authors' contributions}

V.Z and K.Q collected and analyzed survey data, prepared the main manuscript text and prepared figures. All authors reviewed and analyzed the manuscript.

\section{Acknowledgements}

We would like to acknowledge the following physicians for their assistance in data collection: Dr. Jonathan Ailon, Dr. Bilal Asif, Dr. Ben Cadesky, Dr. Luke Devine, Dr. Andrew Duncan, Dr. Edward Etchells, Dr. John Fralick, Dr. David Frost, Dr. Shlok Gupta, Dr. Peter Jaksa, Dr. Moira Kapral, Dr. Dhanjit Litt, Dr. Tara O’Brien, Dr. Lola Oyenubi, Dr. Katherine Plenk, Dr. Laura Walker. 


\section{$\underline{\text { References }}$}

1. Fried TR, Bradley EH, Towle VR, Allore H. Understanding the treatment preferences of seriously ill patients. N Engl J Med. 2002; 346:1061-1066.

2. Sinuff T, Dodek P, You JJ, Barwich D, Tayler C, Downar J, et al. Improving endof-life communication and decision making: The development of a conceptual framework and quality indicators. J Pain Symptom Manage. 2015 Jun 1;49(6):1070-80.

3. Heyland DK, Cook DJ, Rocker GM, Dodek PM, Kutsogiannis DJ, Skrobik Y, et al. Defining priorities for improving end-of-life care in Canada. CMAJ. 2010 Nov $9 ; 182(16)$

4. Fowler R, Hammer M. End-of-life care in Canada. Clin Investig Med. 2013;36(3):127-32.

5. Bernacki RE, Block SD. Communication about serious illness care goals: A review and synthesis of best practices. JAMA Intern Med. 2014;174(12):19942003.

6. Argintaru N, Quinn KL, Chartier LB, Lee J, Hannam P, O'Connor E, et al. Perceived barriers and facilitators to goals of care discussions in the emergency department: A descriptive analysis of the views of emergency medicine physicians and residents. Can J Emerg Med. 2019 Mar 1;21(2):211-8. 
7. Dosanjh S, Barnes J, Bhandari M. Barriers to breaking bad news among medical and surgical residents. Med Educ. 2001;35:197-205.

8. Ahern SP, Doyle TK, Marquis F, Lesk C, Skrobik Y. Critically ill patients and endof-life decision-making: The senior medical resident experience. Adv Heal Sci Educ. 2012;17:121-136.

9. Lamba S, Nagurka R, Zielinski A, Scott SR. Palliative care provision in the emergency department: Barriers reported by emergency physicians. J Palliat Med. 2013 Feb 1;16(2):143-7.

10. Roze Des Ordons A, Kassam A, Simon J. Goals of care conversation teaching in residency - a cross-sectional survey of postgraduate program directors. BMC Med Educ. 2017; 6(2017).

11. Verma AA, Guo Y, Kwan JL, Lapointe-Shaw L, Rawal S, Tang T, et al. Patient characteristics, resource use and outcomes associated with general internal medicine hospital care: the General Medicine Inpatient Initiative (GEMINI) retrospective cohort study. C Open. 2017 Dec 11;5(4):E842-9.

12. You JJ, Downar J, Fowler RA, Lamontagne F, Ma IW, Jayaraman D, et al. Barriers to goals of care discussions with seriously ill hospitalized patients and their families: a multicenter survey of clinicians. JAMA Intern Med. 2015 Apr;175(4): 549-56.

13. Livingston EH, Wislar JS. Minimum response rates for survey research. Vol. 147, Archives of Surgery. 2012. p. 110. 
14. Johnson TP, Wislar JS. Response rates and nonresponse errors in surveys. Vol. 307, JAMA - Journal of the American Medical Association. 2012. p. 1805-6.

15. Tinetti ME, Naik AD, Dodson JA. Moving from disease-centered to patient goalsdirected care for patients with multiple chronic conditions: Patient value-based care. JAMA Cardiology. 2016.

16. Tinetti ME, Fried TR, Boyd CM. Designing health care for the most common chronic condition - Multimorbidity. JAMA - Journal of the American Medical Association. 2012.

17. Ouchi K, Wu M, Medairos R, Grudzen CR, Balsells H, Marcus D, et al. Initiating palliative care consults for advanced dementia patients in the emergency department. J Palliat Med. 2014 Mar 1;17(3):346-50.

18. Quinn KL, Grudzen CR, Smith AK, Detsky AS. Stop that Train! I Want to Get Off: Emergency Care for Patients with Advanced Dementia. Can J Gen Intern Med. $2017 ; 12(1)$.

19. Grudzen CR, Stone SC, Morrison RS. The palliative care model for emergency department patients with advanced illness. J Palliat Med. 2011; 14(8):945-950.

20. Grudzen CR, Hwang U, Cohen JA, Fischman M, Morrison RS. Characteristics of emergency department patients who receive a palliative care consultation. $\mathrm{J}$ Palliat Med. 2012 Apr 1;15(4):396-9. 


\section{Figure 1: Identified Barriers to Goals of Care Discussions in the Emergency}

Department. The differences in the proportion of physicians who responded 'always' and 'never' versus 'disagree' and 'agree' were rank ordered to create a list of the most substantial perceived barriers to having Goals of Care discussions with hospitalized patients cared for in the Emergency Department. * represents $p<0.05 .{ }^{\wedge}$ represents the domain of environment and resources, $\sim$ represents the domain of patient related factors and \# represents the domain of physician responsibility and training.

Communication with patient's healthcare providers * ^

ED working environment is conducive $* \wedge$

Effective prognostic tools exist for patients with non-cancer illness * ^

Challenging once aggressive medical treatment initiated

Sufficient time to communicate with patients/families ^

Prognostic tools more effective for patients with cancer *^

Contacting patient's family/SDM *

Availability of Palliative Care service 24 hours/day* $\wedge$

Conflict between family and health care team *

Patients / families are ready and willing to participate*

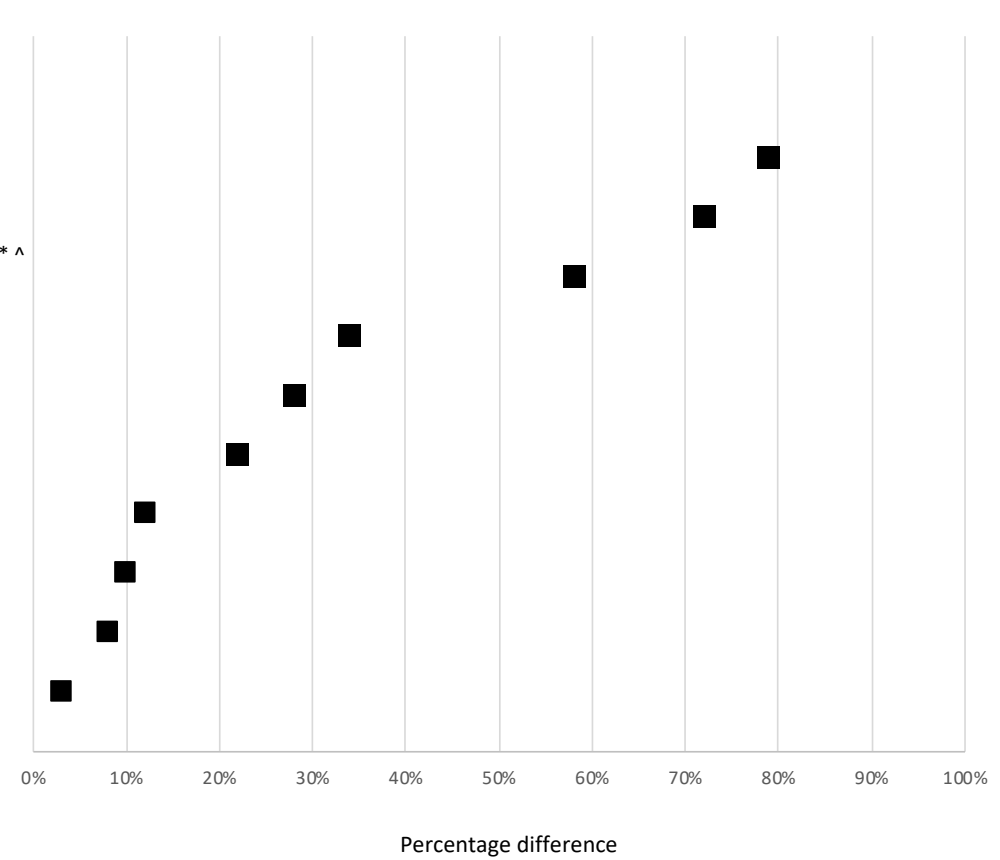


Table 1: Baseline characteristics of hospitalist medicine staff and resident physicians included in the study. General Internal Medicine subspecialty training represents physicians who completed a two year fellowship in the management of patients with complex medical issues and polypharmacy. Critical Care subspecialty training represents physicians who completed a two year fellowship in the management of patients with life-threatening conditions cared for in the intensive care unit.

\begin{tabular}{|l|l|l|}
\hline & \multicolumn{2}{|c|}{$\begin{array}{c}\text { Physician Type } \\
(n=181)\end{array}$} \\
\hline & $\begin{array}{c}\text { Staff Physician } \\
(n=110)\end{array}$ & $\begin{array}{c}\text { Resident Physician } \\
\text { (n=71) }\end{array}$ \\
\hline Female , n (\%) & $31(30)$ & $36(51)$ \\
\hline Years in practise, $n$ (\%) & -- & $25(39)$ \\
\hline 1st year of residency & -- & $18(28)$ \\
\hline 2nd year of residency & -- & $21(33)$ \\
\hline $3^{\text {rd }}$ year of residency & $17(16)$ & -- \\
\hline $0-5$ & $46(43)$ & - \\
\hline 5-10 yrs & & \\
\hline
\end{tabular}




\begin{tabular}{|l|l|l|}
\hline $10-20$ yrs & $25(24)$ & - \\
\hline$>20$ yrs & $18(17)$ & -- \\
\hline Further Subspecialty Training, $n(\%)$ & & \\
\hline General Internal Medicine & $64(84)$ & -- \\
\hline Critical Care & & \\
\hline Other & $5(6)$ & -- \\
\hline
\end{tabular}


Table 2: Representative responses of hospitalist physicians to the prompt, 'What do you find challenging about having Goals of Care discussions in the Emergency

Department?'. Responses were assigned into one of three domains associated with Goals of Care discussions.

Domain

Representative Response

Environment \&

Resources
"The time pressure and the lack of physical space that is private- it is frequently a conversation I unfortunately have in the hallway."
Patient-Related

Factors
"Families come to the hospital for help and for their loved one to get better. Many are not willing to acknowledge decline and inevitable death (perhaps on this admission) in the frail or very elderly."
Physician Responsibility \& Training
"These conversations are most difficult when physicians who have a therapeutic relationship -- and who ought to have provided education around prognosis or futility around some extraordinary level of care (intubation, CPR) -- did not do so. Patients and their families tend to be much less receptive to the newcomer physician in the ED who is the "bearer of bad news"." 


\section{Supplementary}

S1: Internal Medicine Staff Survey

S2: Internal Medicine Resident Survey

S3: Title Page 


\section{Figures}

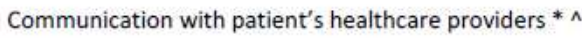

ED working environment is conducive * A

Effective prognostic tools exist for patients with non-cancer illness * $A$

Challenging once aggressive medical treatment initiated

Sufficient time to communicate with patients/families ^

Prognostic tools more effective for patients with cancer * $\wedge$

Contacting patient's family/ SDM *

Availability of Palliative Care service 24 hours/day* ^

Conflict between family and health care team *

Patients / families are ready and willing to participate*

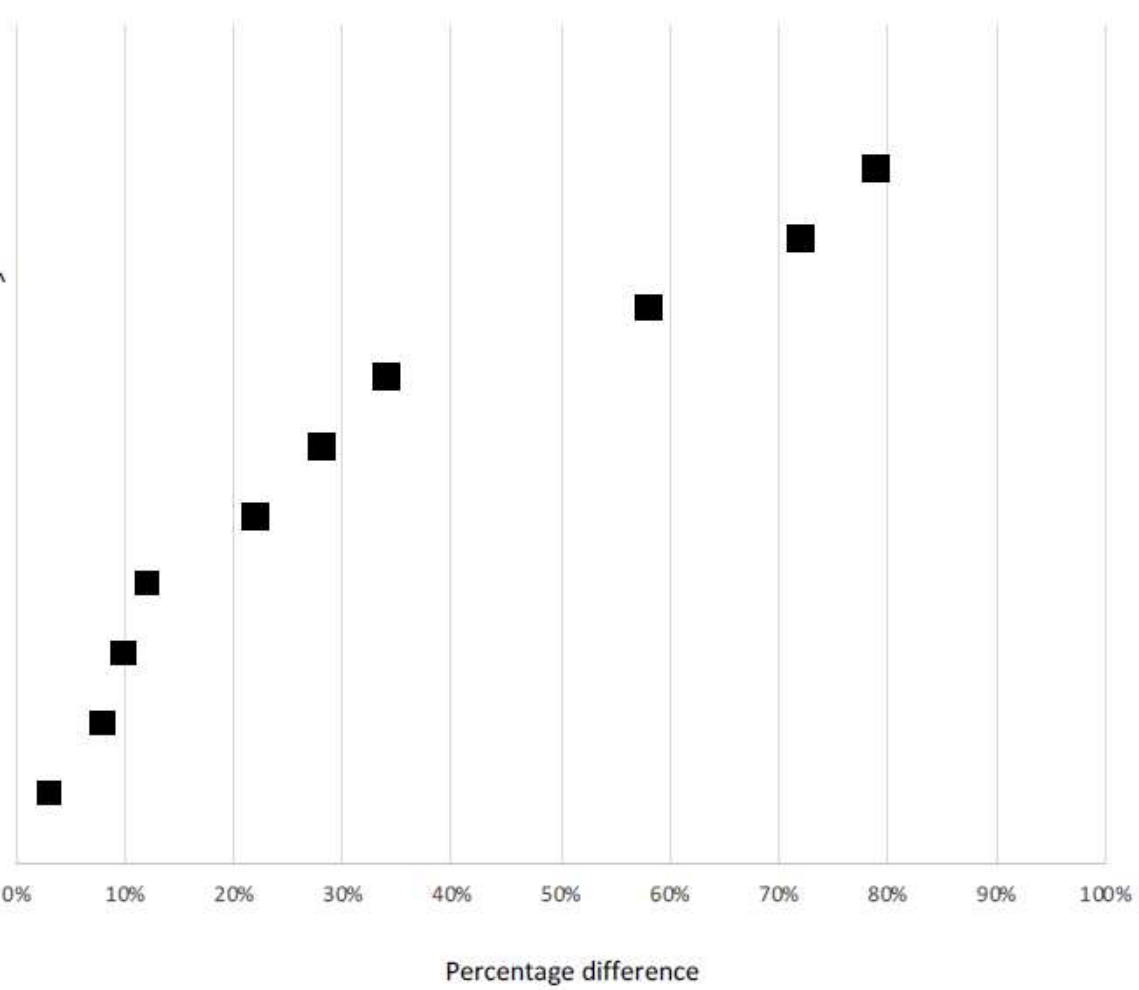

\section{Figure 1}

Identified Barriers to Goals of Care Discussions in the Emergency Department. The differences in the proportion of physicians who responded 'always' and 'never' versus 'disagree' and 'agree' were rank ordered to create a list of the most substantial perceived barriers to having Goals of Care discussions with hospitalized patients cared for in the Emergency Department. * represents $p<0.05 .{ }^{\wedge}$ represents the domain of environment and resources, $\sim$ represents the domain of patient related factors and \# represents the domain of physician responsibility and training.

\section{Supplementary Files}

This is a list of supplementary files associated with this preprint. Click to download.

- SurveyInternalMedicineResident.docx

- SurveyInternalMedicineStaff.docx 